\title{
AN INEQUALITY OF ARAKI-LIEB-THIRRING (VON NEUMANN ALGEBRA CASE)
}

\author{
HIDEKI KOSAKI
}

(Communicated by Paul S. Muhly)

\begin{abstract}
For a trace $\tau$ on a semifinite von Neumann algebra we will prove $\tau\left(\left(b^{1 / 2} a b^{1 / 2}\right)^{r p}\right) \leq \tau\left(\left(b^{r / 2} a^{r} b^{r / 2}\right)^{p}\right)$. Here, $r \geq 1, p>0$, and $a, b$ are positive operators.
\end{abstract}

\section{INTRODUCTION}

In [1] Araki proved the following inequality:

$$
\operatorname{Tr}\left(\left(b^{1 / 2} a b^{1 / 2}\right)^{r p}\right) \leq \operatorname{Tr}\left(\left(b^{r / 2} a^{r} b^{r / 2}\right)^{p}\right), \quad r \geq 1, p>0 .
$$

Here, $a, b$ are positive operators, and $\operatorname{Tr}$ denotes the usual trace for operators on a Hilbert space. This inequality is a generalization of the one due to Lieb and Thirring, and closely related to the Golden-Thompson inequality (see [7, $\S 8])$. In [1] Araki asked whether the same estimate remains valid for a general trace on a semifinite von Neumann algebra. The method in [1] is to obtain a certain majorization of eigenvalues from a related operator-norm inequality (see [4]) based on the classical trick involving antisymmetric tensors. Although this trick is not available for von Neumann algebras, we show that the above inequality remains valid in the setup of general von Neumann algebras (with or without traces). We will use generalized $s$-numbers explained in $[2,3,5]$.

\section{Majorization}

Throughout this article, let $N$ be a semifinite von Neumann algebra with a faithful normal semifinite trace $\tau$. For a $\tau$-measurable operator $x$ (affiliated with $N$ ), we denote the generalized $s$-number by $\mu_{s}(x), s>0$. (See [3] for details.) As in [2] we set

$$
K=\left\{x \in N: \lim _{s \rightarrow \infty} \mu_{s}(x)=0\right\},
$$

which is a two-sided ideal in $N$. For $x \in K$ and $t \in(0, \tau(1))(\tau(1)$ could be $+\infty)$, we set

$$
\Lambda_{t}(x)=\exp \left(\int_{0}^{t} \log \mu_{s}(x) d s\right) .
$$

Received by the editors August 5, 1990.

1980 Mathematics Subject Classification (1985 Revision). Primary 46L10; Secondary 47B15. 
Basic properties of $\Lambda_{t}(\cdot)$ are

$$
\left\{\begin{aligned}
\Lambda_{t}(x) & =\Lambda_{t}\left(x^{*}\right)=\Lambda_{t}(|x|) \\
\Lambda_{t}\left(x^{\alpha}\right) & =\Lambda_{t}(x)^{\alpha} \quad \text { if } \alpha>0 \text { and } x \text { is positive } \\
\Lambda_{t}(x y) & \leq \Lambda_{t}(x) \Lambda_{t}(y)
\end{aligned}\right.
$$

The last inequality is Theorem 2.3, [2] (see [6] for a stronger estimate) and related to the Fuglede-Kadison determinant theory. In the proof of Theorem 5.2 , [2], the following is shown:

$$
\Lambda_{t}\left(|a b|^{2^{n}}\right) \leq \Lambda_{t}\left(a^{2^{n}} b^{2^{n}}\right)
$$

$t>0, n=0,1,2, \ldots$, and $a, b \in K_{+}$. When $n=1$, this means

$$
\Lambda_{t}\left(b^{1 / 2} a b^{1 / 2}\right)=\Lambda_{t}\left(\left|a^{1 / 2} b^{1 / 2}\right|^{2}\right) \leq \Lambda_{t}(a b) .
$$

Although (2) is sufficient for our later purpose, the next stronger result (see [7, Theorem 8.1]) may be of independent interest.

Remark 1. Assume $x, y \in K$. If the product $x y$ is selfadjoint, then we have $\Lambda_{t}(x y) \leq \Lambda_{t}(y x), t>0$.

The following proof is a variant of that of [2, Theorem 5.2]. We may and do assume $\Lambda_{t}(x), \Lambda_{t}(y) \neq 0$ (thanks to $\Lambda_{t}(x y) \leq \Lambda_{t}(x) \Lambda_{t}(y)$ ). For each $n \in \mathbb{N}_{+}$, we estimate

$$
\begin{aligned}
\Lambda_{t}(x y)^{2 n} & =\Lambda_{t}\left(|x y|^{2 n}\right) \\
& =\Lambda_{t}\left((x y)^{2 n}\right) \quad(\text { since } x y \text { is selfadjoint }) \\
& =\Lambda_{t}\left(x(y x)^{2 n-1} y\right) \leq \Lambda_{t}(x) \Lambda_{t}(y x)^{2 n-1} \Lambda_{t}(y) .
\end{aligned}
$$

Taking the $2 n$th roots of the both sides and then letting $n$ go to $+\infty$, we obtain the desired result.

Let us return to (1). Replacing $a, b$ by $a^{1 / 2^{n}}, b^{1 / 2^{n}}$ respectively, we get

$$
\Lambda_{t}\left(a^{1 / 2^{n}} b^{1 / 2^{n}}\right) \leq \Lambda_{t}(a b)^{1 / 2^{n}} .
$$

We would like to generalize this to

$$
\Lambda_{t}\left(a^{r} b^{r}\right) \leq \Lambda_{t}(a b)^{r}
$$

for $r \in(0,1]$ (and $a, b \in K_{+}$). For this purpose we prove the following claim: If the inequality is valid for $r=\alpha$ and $r=\beta, 0<\beta<\alpha \leq 1$, then so is the case for $r=(\alpha+\beta) / 2$. In fact, we estimate

$$
\begin{aligned}
\Lambda_{t}\left(a^{\frac{\alpha+\beta}{2}} b^{\frac{\alpha+\beta}{2}}\right)^{2} & =\Lambda_{t}\left(b^{\frac{\alpha+\beta}{2}} a^{\alpha+\beta} b^{\frac{\alpha+\beta}{2}}\right)=\Lambda_{t}\left(b^{\frac{\alpha-\beta}{2}}\left(b^{\beta} a^{\alpha+\beta} b^{\beta}\right) b^{\frac{\alpha-\beta}{2}}\right) \\
& \leq \Lambda_{t}\left(\left(b^{\beta} a^{\alpha+\beta} b^{\beta}\right) b^{\alpha-\beta}\right) \quad(\text { by }(2) \text { or Remark 1) } \\
& =\Lambda_{t}\left(\left(b^{\beta} a^{\beta}\right)\left(a^{\alpha} b^{\alpha}\right)\right) \leq \Lambda_{t}\left(b^{\beta} a^{\beta}\right) \Lambda_{t}\left(a^{\alpha} b^{\alpha}\right) \\
& =\Lambda_{t}\left(a^{\alpha} b^{\alpha}\right) \Lambda_{t}\left(a^{\beta} b^{\beta}\right) \leq \Lambda_{t}(a b)^{\alpha} \Lambda_{t}(a b)^{\beta} \quad \text { (by the assumption). }
\end{aligned}
$$

By taking the square roots, we are done.

Hence, (3) is valid for $r$ in the dense subset in $(0,1]$. Replacing $a^{r}, b^{r}$ by $a, b$ respectively, we observe:

$$
\Lambda_{t}\left(|a b|^{r}\right) \leq \Lambda_{t}\left(a^{r} b^{r}\right) ; \quad t>0, a, b \in K_{+}
$$


for $r$ in the dense subset in $[1,+\infty)$. We postpone generalizing these inequalities for an arbitrary $r$ (see the paragraph after Theorem 2).

\section{APPROXIMATION}

Let $f$ be a continuous increasing function on $\mathbb{R}_{+}$such that $f(0)=0$ and $t \rightarrow f\left(e^{t}\right)$ is convex. By the Weyl majorization theorem (see [2, Corollary 4.2]), from (4) we get

$$
\int_{0}^{t} f\left(\mu_{s}\left(|a b|^{r}\right)\right) d s \leq \int_{0}^{t} f\left(\mu_{s}\left(a^{r} b^{r}\right)\right) d s, \quad t>0 .
$$

To show (5) for an arbitrary $r \geq 1$, we choose a sequence $\left\{r_{n}\right\}$ for which (4) (and hence (5)) is valid. Since (for each $s$ ) the map: $x \rightarrow \mu_{s}(x)$ is normcontinuous [2, Proposition 1.6 (iii)], the standard argument on norm convergence and the dominated convergence theorem shows

$$
\left\{\begin{array}{l}
\int_{0}^{t} f\left(\mu_{s}\left(|a b|^{r}\right)\right) d s=\lim _{n \rightarrow \infty} \int_{0}^{t} f\left(\mu_{s}\left(|a b|^{r_{n}}\right)\right) d s, \\
\int_{0}^{t} f\left(\mu_{s}\left(a^{r} b^{r}\right)\right) d s=\lim _{n \rightarrow \infty} \int_{0}^{t} f\left(\mu_{s}\left(a^{r_{n}} b^{r_{n}}\right)\right) d s .
\end{array}\right.
$$

Hence (5) is valid for an arbitrary $r \geq 1$ (and $a, b \in K_{+}$).

Next we would like to generalize $(5)$ for positive $\tau$-measurable operators $a, b$ satisfying $\lim _{s \rightarrow \infty} \mu_{s}(a)=\lim _{s \rightarrow \infty} \mu_{s}(b)=0$. Let $a=\int_{0}^{\infty} \lambda d e_{\lambda}$ and $b=\int_{0}^{\infty} \lambda d f_{\lambda}$ be the spectral decompositions. We set

$$
a_{n}=\int_{n^{-1}}^{n} \lambda d e_{\lambda}, \quad b_{n}=\int_{n^{-1}}^{n} \lambda d f_{\lambda} \quad\left(n \in \mathbb{N}_{+}\right) .
$$

(See [3, Proposition 3.2].) Fixing $m \in \mathbb{N}_{+}$, we estimate

$$
\begin{aligned}
\int_{0}^{t} f\left(\mu_{s}\left(\left|a b_{m}\right|^{r}\right)\right) d s= & \int_{0}^{t} f\left(\mu_{s}\left(b_{m} a^{2} b_{m}\right)^{r / 2}\right) d s \\
= & \sup _{n} \int_{0}^{t} f\left(\mu_{s}\left(b_{m} a_{n}^{2} b_{m}\right)^{r / 2}\right) d s \\
& \left(\begin{array}{l}
\text { Since } a_{n}^{2} \uparrow a^{2} \text { in measure, [3, Lemma 3.4] shows } \\
\left.\mu_{s}\left(b_{m} a_{n}^{2} b_{m}\right) \uparrow \mu_{s}\left(b_{m} a^{2} b_{m}\right) \text {. Hence the result follows }\right) \\
\text { from the monotone convergence theorem. }
\end{array}\right. \\
= & \sup _{n} \int_{0}^{t} f\left(\mu_{s}\left(\left|a_{n} b_{m}\right|^{r}\right)\right) d s \\
\leq & \sup _{n} \int_{0}^{t} f\left(\mu_{s}\left(a_{n}^{r} b_{m}^{r}\right)\right) d s \quad\left(\text { since } a_{n}, b_{m} \in K_{+}\right) \\
= & \sup _{n} \int_{0}^{t} f\left(\mu_{s}\left(b_{m}^{r} a_{n}^{2 r} b_{m}^{r}\right)^{1 / 2}\right) d s \\
= & \int_{0}^{t} f\left(\mu_{s}\left(b_{m}^{r} a^{2 r} b_{m}^{r}\right)^{1 / 2}\right) d s \\
= & \int_{0}^{t} f\left(\mu_{s}\left(a^{r} b_{m}^{r}\right)\right) d s .
\end{aligned}
$$


Notice $\mu_{s}\left(\left|a b_{m}\right|^{r}\right)=\mu_{s}\left(a b_{m}\right)^{r}=\mu_{s}\left(b_{m} a\right)^{r}=\mu_{s}\left(\left|b_{m} a\right|^{r}\right)$ and $\mu_{s}\left(a^{r} b_{m}^{r}\right)=\mu_{s}\left(b_{m}^{r} a^{r}\right)$. Hence, when $m$ goes to $+\infty$, we can use the monotone convergence as in the preceding argument and get (5) for $a, b$ in the wider class.

\section{MAin Results}

In the $\S \S 1,2$ we have shown

Theorem 2 [1, Theorem 2]. Let $r \geq 1$ and $f$ be a continuous increasing function on $\mathbb{R}_{+}$such that $f(0)=0$ and $t \rightarrow f\left(e^{t}\right)$ is convex. For positive $\tau$-measurable operators $a, b$ satisfying $\lim _{s \rightarrow \infty} \mu_{s}(a)=\lim _{s \rightarrow \infty} \mu_{s}(b)=0$, we have

$$
\int_{0}^{t} f\left(\mu_{s}\left(|a b|^{r}\right)\right) d s \leq \int_{0}^{t} f\left(\mu_{s}\left(a^{r} b^{r}\right)\right) d s, \quad t>0 .
$$

When $f(t)=t^{p}, p>0$, the theorem means

$$
\int_{0}^{t} \mu_{s}\left(|a b|^{r p}\right) d s \leq \int_{0}^{t} \mu_{s}\left(\left|a^{r} b^{r}\right|^{p}\right) d s, \quad t>0 .
$$

For a $\tau$-measurable operator $x$ satisfying the "Lorentz space condition" $\left(\mu_{s}(x)\right.$ $\leq C s^{-\alpha}$ for some $\left.C, \alpha>0\right)$, the expression $\Lambda_{t}(x)=\exp \left(\int_{0}^{t} \log \mu_{s}(x) d s\right)$ still makes sense (see $[3$, p. 286]). For such positive operators $a, b,(6)$ obviously implies

$$
\left\{\int_{0}^{t} \mu_{s}\left(|a b|^{r}\right)^{p} \frac{d s}{t}\right\}^{1 / p} \leq\left\{\int_{0}^{t} \mu_{s}\left(a^{r} b^{r}\right)^{p} \frac{d s}{t}\right\}^{1 / p} .
$$

Letting $p \downarrow 0$ (see [3, p. 288]), we conclude that (4) is valid for an arbitrary $r \in[1,+\infty)$, or equivalently, (3) is valid for an arbitrary $r \in(0,1]$.

Letting $t \uparrow+\infty$ in (6) (see [3, Corollary 2.8]), we get the following (semifinite) von Neumann algebra version of the inequality in the introduction:

Corollary 3. For positive operators $a, b$ in the previous theorem we have

$$
\tau\left(|a b|^{r p}\right) \leq \tau\left(\left|a^{r} b^{r}\right|^{p}\right) ; \quad r \geq 1, p>0 .
$$

Let $\|x\|_{p}=\tau\left(|x|^{p}\right)^{1 / p}, p>0$, be the $L^{p}$ (quasi-)norm. Then the corollary means $\left\||a b|^{r}\right\|_{p} \leq\left\|a^{r} b^{r}\right\|_{p}$. Let $M$ be a general (not necessarily semifinite) von Neumann algebra. We have (for example Haagerup) $L^{p}$-spaces $L^{p}(M)$, $p>0$, and $\|\cdot\|_{p}$ still makes sense. (See $[3,1.6 ; 5]$ for quick introduction to the subject.)

Theorem 4. Let $r \geq 1$ and $a, b$ be positive elements in $L^{p_{1} r}(M)$ and $L^{p_{2} r}(M)$ $\left(p_{i}>0\right)$ respectively. For $p>0$ satisfying $p^{-1}=p_{1}^{-1}+p_{2}^{-1}$ (hence ab $\in L^{p r}(M)$ and $\left.|a b|^{r}, a^{r} b^{r} \in L^{p}(M)\right)$ we have

$$
\left\||a b|^{r}\right\|_{p} \leq\left\|a^{r} b^{r}\right\|_{p} .
$$

Proof. We will use the trick used in the appendix to [5]. Let $N$ be the crossed product $M \rtimes_{\sigma} \mathbb{R}$ relative to a modular automorphism group, and $\tau$ be the canonical trace on $N$ scaled (in the usual way) by the dual action. From the definition, $a, b$ are $\tau$-measurable operators (affiliated with $N$ ), and [3, Lemma 4.8] says

$$
\left\{\begin{array}{l}
\mu_{s}(a b)=\|a b\|_{p r} s^{-1 / p r} \\
\mu_{s}\left(a^{r} b^{r}\right)=\left\|a^{r} b^{r}\right\|_{p} s^{-1 / p}
\end{array}\right.
$$


Therefore, (6) (with $t=1$ and $p / 2$ instead of $p$ ) implies that

$$
\int_{0}^{1}\left(\|a b\|_{p r} s^{-1 / p r}\right)^{p r / 2} d s \leq \int_{0}^{1}\left(\left\|a^{r} b^{r}\right\|_{p} s^{-1 / p}\right)^{p / 2} d s .
$$

Evaluating the integrals, we obtain $2\|a b\|_{p r}^{p r / 2} \leq 2\left\|a^{r} b^{r}\right\|_{p}^{p / 2}$, that is, $\|a b\|_{p r}^{r} \leq$ $\left\|a^{r} b^{r}\right\|_{p}$. However, the left side is obviously equal to $\left\||a b|^{r}\right\|_{p}$. Q.E.D.

\section{REFERENCES}

1. H. Araki, On an inequality of Lieb and Thirring, Lett. Math. Phys. 19 (1990), 167-170.

2. T. Fack, Sur la notion de valeur caractéristique, J. Operator Theory 7 (1982), 307-333.

3. T. Fack and H. Kosaki, Generalized s-numbers of $\tau$-measurable operators, Pacific J. Math. 123 (1986), 269-300.

4. T. Furuta, Norm inequalities equivalent to Löwner-Heinz theorem, Reviews in Math. Phys. 1 (1989), 135-137.

5. H. Kosaki, On the continuity of the map $\phi \rightarrow|\phi|$ from the predual of a $W^{*}$-algebra, J. Funct. Anal. 59 (1984), 123-131.

6. Y. Nakamura, An inequality for generalized s-numbers, Integral Equations Operator Theory 10 (1987), 140-145.

7. B. Simon, Trace ideals and their applications, Cambridge Univ. Press, 1979.

Department of Mathematics, College of General Education, Kyushu University, FUKUOKA 810, JAPAN 